\title{
General Psychiatry Microglia activation in the offspring of prenatal Poly I: C exposed rats: a PET imaging and immunohistochemistry study
}

\author{
Xue Li, ${ }^{1}$ Xin Tian, ${ }^{1}$ Luxian Lv, ${ }^{2,3}$ Gangrui Hei, ${ }^{1}$ Xufeng Huang, ${ }^{4}$ Xiaoduo Fan, ${ }^{5}$ \\ Jinming Zhang, ${ }^{6}$ Jianjiang Zhang, ${ }^{1}$ Lijuan Pang, ${ }^{1}$ Xueqin Song ${ }^{1}$
}

To cite: Li X, Tian X, Lv L, et al. Microglia activation in the offspring of prenatal Poly I: C exposed rats: a PET imaging and immunohistochemistry study. General Psychiatry 2018;31:e000006. doi:10.1136/ gpsych-2018-000006

$\mathrm{XL}$ and $\mathrm{XT}$ contributed equally.

Received 12 June 2017

Revised 27 June 2017

Accepted 30 December 2017

Check for updates

(C) Author(s) (or their employer(s)) 2018 . Re-use permitted under CC BY-NC. No commercial re-use. See rights and permissions. Published by BMJ.

Department of Nuclear Medicine, Chinese PLA General Hospital, Beijing, China

${ }^{1}$ The First Affiliated Hospital/ Zhengzhou University,

Zhengzhou, China

${ }^{2}$ Henan Province Biological Psychiatry Key Laboratory, Xinxiang Medical University, Xinxiang, China

${ }^{3}$ Henan Province Mental Hospital, The Second Affiliated Hospital, Xinxiang Medical University, Xinxiang, China ${ }^{4}$ School of Medicine and IHMRI, University of Wollongong,

Wollongong, New South Wales, Australia

${ }^{5}$ UMass Memorial Medical

Center, University of

Massachusetts Medical School, Worcester, Massachusetts, USA

${ }^{6}$ Department of Nuclear

Medicine, Chinese PLA General Hospital, Beijing, China

Correspondence to

Xueqin Song; sxqzh\|॥126.com

\section{ABSTRACT}

Background The well-known 'pyrotherapy' of Julius Wagner-Jauregg might be the beginning of the study on the immunological concepts of schizophrenia. As the primary immune effector cells in the brain, microglia play a pivotal role in neuroinflammatory processes. Maternal viral infection during pregnancy is associated with an increased risk for psychiatric disorders with presumed neurodevelopmental origin, including autism spectrum disorders and schizophrenia. The present study was to quantify microglia activation in vivo in the mature offspring of rats exposed to polyriboinosinic-polyribocytidilicacid (Poly I:C) during pregnancy using ${ }^{11} \mathrm{C}$-PK11195 positron emission tomography (PET) and immunohistochemistry. Objective The study aimed to quantify microglia activation in vivo in the prefrontal cortex and hippocampus in mature offspring of prenatal Poly I:C exposed rats.

Methods Offspring of Poly l:C-treated dams were the model group, offspring of saline-treated dams were the control group. Behavioural test for two groups was taken by spontaneous activity, prepulse inhibition (PPI) and latent inhibition (LI) test (including active avoidance conditioning task and passive avoidance conditioning task). Randomly selected successful model rats were assessed by behavioural test in the model group and control group rats. ${ }^{11} \mathrm{C}-\mathrm{PK} 11195$ micro-PET/CT and immunohistochemistry were performed on the selected rats to measure microglia activation.

Results The treatment group showed hyperlocomotion and deficits in PPI and LI compared with the control group. The treatment group also showed an increased ${ }^{11} \mathrm{C}$-PK11195 uptake ratio in the prefrontal cortex $(t=-3.990, p=0.003)$ and hippocampus $(t=-4.462$, $p=0.001)$. The number of activated microglia cells was significantly higher in the treatment group than in the control group (hippocampus: $t=8.204, p<0.001$; prefrontal: $t=6.995, p<0.001)$. Within the treatment group, there were significant correlations between the behavioural parameters and the activation of microglia as measured by PET and immunohistochemistry.

Conclusions The present study demonstrated microglia activation in vivo in the prefrontal cortex and hippocampus in mature offspring of prenatal Poly I:C exposed rats. This study suggests that microglia activation may play a possible or potential role in the pathogenesis of schizophrenia.

\section{INTRODUCTION}

The well-known 'pyrotherapy' of Julius Wagner-Jauregg might be the beginning of the study on the immunological concepts of schizophrenia (SCZ). Our previous studies reported elevated serum levels of interleukin-1 $\beta$ (IL-1 $\beta$ ) and tumour necrosis factor (TNF)- $\alpha$ (TNF- $\alpha$ ), increased nuclear factor-кB (NF-KB) activation and its mRNA expression, and activated Th17 cells in peripheral blood mononuclear cells in patients with first-episode SCZ. ${ }^{1}$ A human postmortem study has shown increased cytokine levels in the brains of patients with SCZ. ${ }^{2}$ As the primary immune effector cells in the brain, microglia play a pivotal role in neuroinflammatory processes. ${ }^{3}$

Maternal viral infection during pregnancy is associated with an increased risk of psychiatric disorders with presumed neurodevelopmental origin, including autism spectrum disorders and SCZ. ${ }^{4}$ Bilbo and colleauges have hypothesised that subsets of microglia are permanently maintained in an activated or primed state into adulthood as a consequence of prenatal infection, and that a subsequent immune challenge in adulthood can cause exaggerated levels of cytokines from these already primed microglia. ${ }^{56}$

Recently, a great deal of interest has been centred on the establishment of neurodevelopmental rodent models in which the basic experimental manipulation takes the form of prenatal exposure to infection and/or immune activation. ${ }^{7}$ In addition to the influenza infection animal model, ${ }^{8}$ an animal model has been increasingly used in SCZ research: offspring of female mice that were administered viral mimetic polyriboinosinic-polyribocytidilic acid (Poly I:C) during pregnancy. Those offspring reportedly show changes in brain morphology, physiology, chemistry as well as behaviours 
that are similar to changes observed in patients with SCZ. ${ }^{910}$ Specifically, prenatal Poly I:C treatment on the gestation date (GD) 9 leads to a pathological profile in the offspring characterised by suppression in exploratory behaviour, abnormalities in selective associative learning in the form of latent inhibition (LI) disruption and abolition of the US pre-exposure effect, impairments in sensor motor gating in the form of reduced prepulse inhibition (PPI), enhanced sensitivity to the indirect dopamine receptor agonist amphetamine and deficiency in spatial working memory when the demand on temporal retention is high. ${ }^{11} 12$ The findings from our group showed that the serum levels of cytokines (TNF- $\alpha$ and IL-10) in the offspring increased after an injection of Poly I:C in rats during early pregnancy, and the NF-кB inhibitor pyrrolidine dithiocarbamate suppressed the maternal immune response induced by Poly I:C and partially prevented the behavioural changes in the offspring that mimicked neurodevelopmental disorders.

It is well known that microglia activation can be visualised in vivo using ${ }^{11}$ C-PK11195 (PK11195) positron emission tomography (PET). ${ }^{13}$ The present study was to quantify microglia activation in vivo in the mature offspring of rats exposed to Poly I:C during pregnancy using ${ }^{11}$ C-PK11195 PET and immunohistochemistry. The brain regions of interest (ROI) included the prefrontal cortex and hippocampus, which have been frequently reported to be involved in the pathogenesis of SCZ.

\section{METHODS AND MATERIALS \\ Animals}

Female and male-specific pathogen-free colony SpragueDawley (SD) rats (10-12 weeks old) were obtained from the Laboratory Animal Center of Zhengzhou University (Zhengzhou, Henan, China) and individually housed in a temperature and humidity-controlled environment with a 12-hour light/12-hour dark cycle (lights on at 07:00). Food and tap water were available ad libitum. All animal experiments in the present study were approved by the Animal Care and Use Committee at Xinxiang Medical University, Xinxiang, China, where the experiment was carried out. After 2 weeks of acclimatisation, male and female rats were colocated overnight. Male rats were removed in the morning of the next day. The breeding was stopped until 18 pregnant rats were accomplished. The successful copulation verified by the presence of a vaginal plug was considered GD 1.

\section{Modelling method}

Eighteen pregnant rats were randomly divided into two groups. On GD 9, all pregnant dams were weighed, and received a single caudal vein injection of Poly I:C or saline. The treatment group was defined as the offspring of pregnant dams $(\mathrm{n}=9)$ that received a $10 \mathrm{mg} / \mathrm{kg}$ Poly I:C injection ${ }^{15} 16$; the control group included the offspring of pregnant dams $(n=9)$ that received a $10 \mathrm{mg} / \mathrm{kg}$ saline injection. The injections were performed between 09:00
Table 1 Sample size of each group and the sequence of behavioural testing

\begin{tabular}{|c|c|c|}
\hline Experiments & Control group & Treatment group \\
\hline Locomotor activity & 10 우 $90^{\lambda}$ & $10+100^{\lambda}$ \\
\hline Passive avoidance & $9 \circ 9 \hat{O}^{-1}$ & $11+10{ }^{\lambda}$ \\
\hline $\begin{array}{l}\text { Active avoidance } \\
\text { procedure }\end{array}$ & $10 \uparrow 10{ }^{\lambda}$ & $9910 \hat{0}$ \\
\hline Prepulse inhibition & 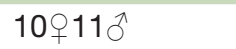 & $10 \circ 9 \widehat{0}$ \\
\hline
\end{tabular}

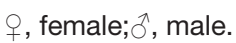

and 10:00 to minimise the variability associated with diurnal rhythms.

\section{Behavioural assessment}

The offspring were weaned at postnatal day 21. Four offspring were housed to a cage by sex and litter, and maintained undisturbed until 8 weeks of age. The gender ratio of the treatment group and control group was approximately 1:1. One of each littermate was randomly selected for behavioural testing. The number of subjects employed in the behavioural testing is summarised in table 1 .

\section{Locomotor activity}

An open-field test was used to assess locomotor activity in a $100 \mathrm{~cm} \times 100 \mathrm{~cm} \times 30 \mathrm{~cm}$ box. Data collection was performed by the SMART video tracking program (Panlab, Spain). To begin testing, the animals were gently placed in the centre of the area, and allowed to explore for $30 \mathrm{~min}$. Rats were tested for $60 \mathrm{~min}$ and data were captured.

\section{Latent inhibition}

LI included both passive and active avoidance tasks. ${ }^{17}$ The passive avoidance conditioning task was carried out over 2 days. On the training day, each rat was placed in the lighted compartment, facing away from the dark compartment. After $5 \mathrm{~s}$, the door was lifted. As soon as the rat entered the dark compartment with all four paws, the guillotine door was closed. The latency from the time the door was lifted to the time the rat entered the compartment was recorded as T1. A foot shock $(0.5 \mathrm{~mA}$, $2 \mathrm{~s}$ duration) was delivered $3 \mathrm{~s}$ after the door was closed. On the test day (24 hours after training), the same experiment procedure was followed as on the training day. The latency from the time the door was lifted to the time the rat entered the compartment was recorded as T2. The rat then returned to the home cage. $T$ was calculated as the difference between $\mathrm{T} 1$ and $\mathrm{T} 2$.

The active avoidance task is a fear-motivated associative avoidance test using an electric current as a source of punishment. In our study, the active avoidance conditioning was carried out in a two-way shuttle box apparatus (Panlab). The subject acclimated to the training box for 10 min before the electric bulb $(10 \mathrm{~W})$ (unconditioned stimulus) was turned on in the compartment where the rat was located. Thirty seconds later, an electric current $(0.3 \mathrm{~mA}, 5 \mathrm{~s})$ (conditioned stimulus) was delivered to 

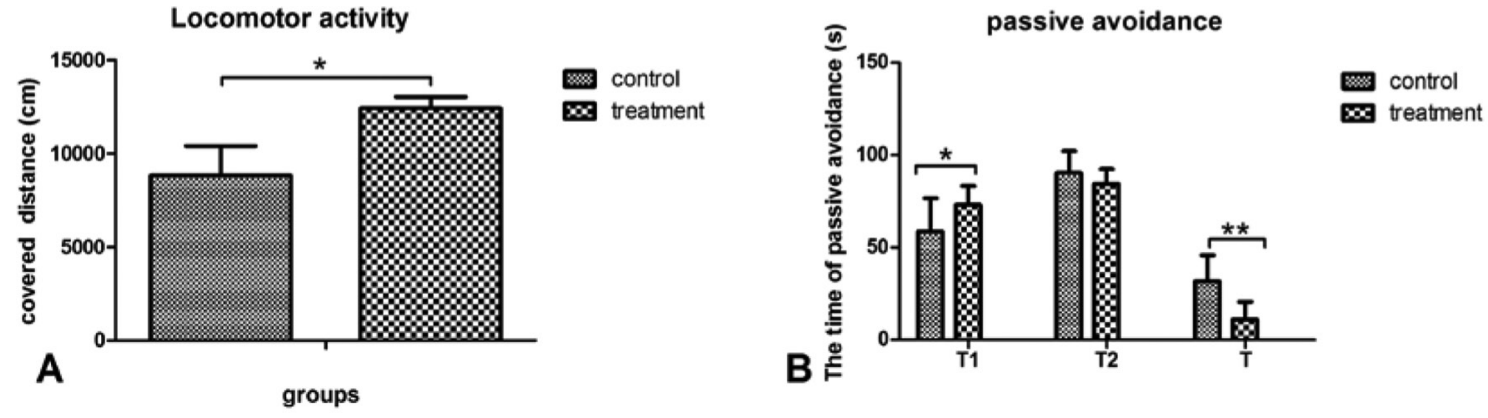

the grid floor of the compartment. The electric current and the light were turned off simultaneously after the rat was transferred into a different compartment. Each rat received 100 trials per day for five consecutive days. ${ }^{18}$ The rate of active avoidance conditioned response was calculated as the total number of conditioned responses $/ 500$. A higher value indicated better learning and memory.

\section{Prepulse inhibition}

PPI is the normal suppression of a startle response when a low-intensity stimulus, which elicits little or no behavioural response, immediately precedes an unexpected stronger startling stimulus. The amount of PPI is expressed as the percentage decrease in the amplitude of the startle response caused by the presentation of the prepulse.

All test sessions were performed in a single chamber startle apparatus (QMC-I, Kunming Institute of Zoology, Chinese Academy of Sciences, China). ${ }^{17}$ One rat was tested during each experimental session. In the test session, the rats were acclimated in the testing cylinder for $5 \mathrm{~min}$, during which time the rats received background noise of only $70 \mathrm{~dB}$ sound pressure level (SPL). The test began with six trials of a pulse-alone startle stimulus, consisting of a $40 \mathrm{~ms}$ burst of white noise of $120 \mathrm{~dB}$ SPL. The session continued with 20 randomised trials, which included five trials of a pulse-alone stimulus and five trials of each of the three types of prepulse startles. A prepulse startle consisted of a prepulse (white noise at 2 , 4 or $8 \mathrm{~dB}$ SPL above the $70 \mathrm{~dB}$ SPL background), a 100 ms interval and a startle pulse (40 ms, $120 \mathrm{~dB}$ SPL white noise). The interval length between the 20 randomised trials varied randomly from 8 to $23 \mathrm{~s}$ with an average of 15 s. Startle responses were measured with the program developed by Kunming Institute of Zoology, Chinese Academy of Sciences, China. The amount of PPI was expressed as the percentage decrease in the amplitude of the startle response caused by the presentation of the prepulse. The amplitude of the startle response without a prepulse is $\mathrm{p}$. When a weak stimulus is given prior to the startle reflex stimulus, the amplitude for the startle response is pp. The percentage of PPI for each rat was calculated as $(1-\mathrm{pp} / \mathrm{p}) \times 100$, which is proportional to the inhibitory effect of PPI. Using this description of PPI, a high degree of sensory motor gating is reflected in a high $\%$ PPI value, whereas a lower or no gating results in a small or negative \% PPI value.

\section{Micro-PET/CT scan}

${ }^{11} \mathrm{C}$-PK11195 was synthesised from the precursors ${ }^{11} \mathrm{C}$-Triflate-CH3 (ABX Express, Germany) and PK11195, with a radiochemical purity of more than $98 \%$, pharmaceutical specifications: specific activity $62.5 \mathrm{TBq} / \mathrm{mol}$.

Rats were anaesthetised for the duration of the scan session with $5 \%$ isoflurane using a system built in-house, which combines the fixed output of a single vaporizer (3\% oxygen) in variable ratios with pure oxygen so that the isoflurane concentration for each rat was individually adjustable. Each rat was injected with $1 \mathrm{mCi}^{11} \mathrm{C}$-PK11195 through the caudal vein. Each rat was scanned for $30 \mathrm{~min}$ with its brain centred in the axial and transaxial fields of view (Explore VISTA Micro-PET/CT scanner from GE Healthcare, USA). The image data were sorted into 
three-dimensional sinograms, followed by attenuation and scatter corrections, Fourier rebinding and two-dimensional ordered-subset expectation maximisation reconstruction with two iterations and 50 subsets. The image pixel size was $0.385 \times 0.385 \times 0.385 \mathrm{~mm}$. For semiquantitative evaluation, the maximum uptake values were automatically measured three times in the ROIs (hippocampus and prefrontal cortex) and the uptake ratio was calculated. ${ }^{19}$

\section{Immunohistochemistry}

Rats were deeply anaesthetised and sacrificed by cervical dislocation. Brains were removed, fixed in $4 \%$ paraformaldehyde for 4-6 hours, dehydrated with $30 \%$ sucrose in phosphate-buffered saline (PBS) overnight, embedded with optimum cutting temperature compound, cut into $10 \mu \mathrm{m}$ thick sections serially using a freezing microtome (CM1950, Leica, Germany), mounted onto superfrost plus glass slides and stored at $-70^{\circ} \mathrm{C}$ for immunohistochemistry. Six sets of serial sections were obtained from each brain, and one of them was used for immunostaining.

Immunohistochemistry was performed as previously described. ${ }^{20}$ After being washed in PBS, endogenous peroxides were blocked by incubation in $0.3 \%$ hydrogen peroxide in $50 \%$ methanol in distilled water for 30 min. Sections were washed in PBS and preincubated in a blocking solution containing $5 \%$ bovine serum albumin for $15 \mathrm{~min}$ at room temperature. Then sections were incubated in a humidified chamber with the rabbit primary antibody against CD11b (1:100; Boster Biological Engineering, China) in PBS overnight at $4^{\circ} \mathrm{C}$. After several washes in PBS, sections were incubated with biotinylated goat anti-rabbit secondary antibody (1:100; Boster Biological Engineering) for $60 \mathrm{~min}$ at room temperature, followed by incubation with the streptavidin-biotin-peroxidase complex method (Boster Biological Engineering). Following the PBS rinsing, the antigen-antibody complexes were visualised using diaminobenzidine (Boster Biological Engineering) as the chromogen. The immunohistochemical controls were performed as above, but with the omission of the primary antibodies. Eight sections were selected randomly from the hippocampus and prefrontal cortex in both groups. Six images were taken from each section using $\times 10$ magnification randomly. Images were captured using a microscope (DM1000, Leica) and the DM2000 Image Acquisition and Analysis System. Image-Pro Plus software (V.6.0, Media Cybernetics, Silver Spring, MD, USA) was used for statistical analysis. Optical density (OD) value was used to quantify the $\mathrm{CD} 11 \mathrm{~b}$ positive cytoplasm. The $\mathrm{OD}$ values were positively correlated with the strength degree of the positive expressed area. OD value=integrated optical density/positive results area.

\section{Statistical analysis}

All analyses were performed using the Statistical Package for the Social Sciences (SPSS V.20.0). Quantitative variables in group comparisons were analysed using the independent t-test. Sex differences were analysed using the independent $\mathrm{X}^{2}$ test. Pearson correlation was used to examine the relationships among behavioural, immunohistochemistry and micro-PET/CT measures. Because there was no significant sex difference in any of the preliminary analyses performed, the male and female subjects were collapsed to enhance statistical power. Statistical significance was set at $\mathrm{p}<0.05$ (two tailed).
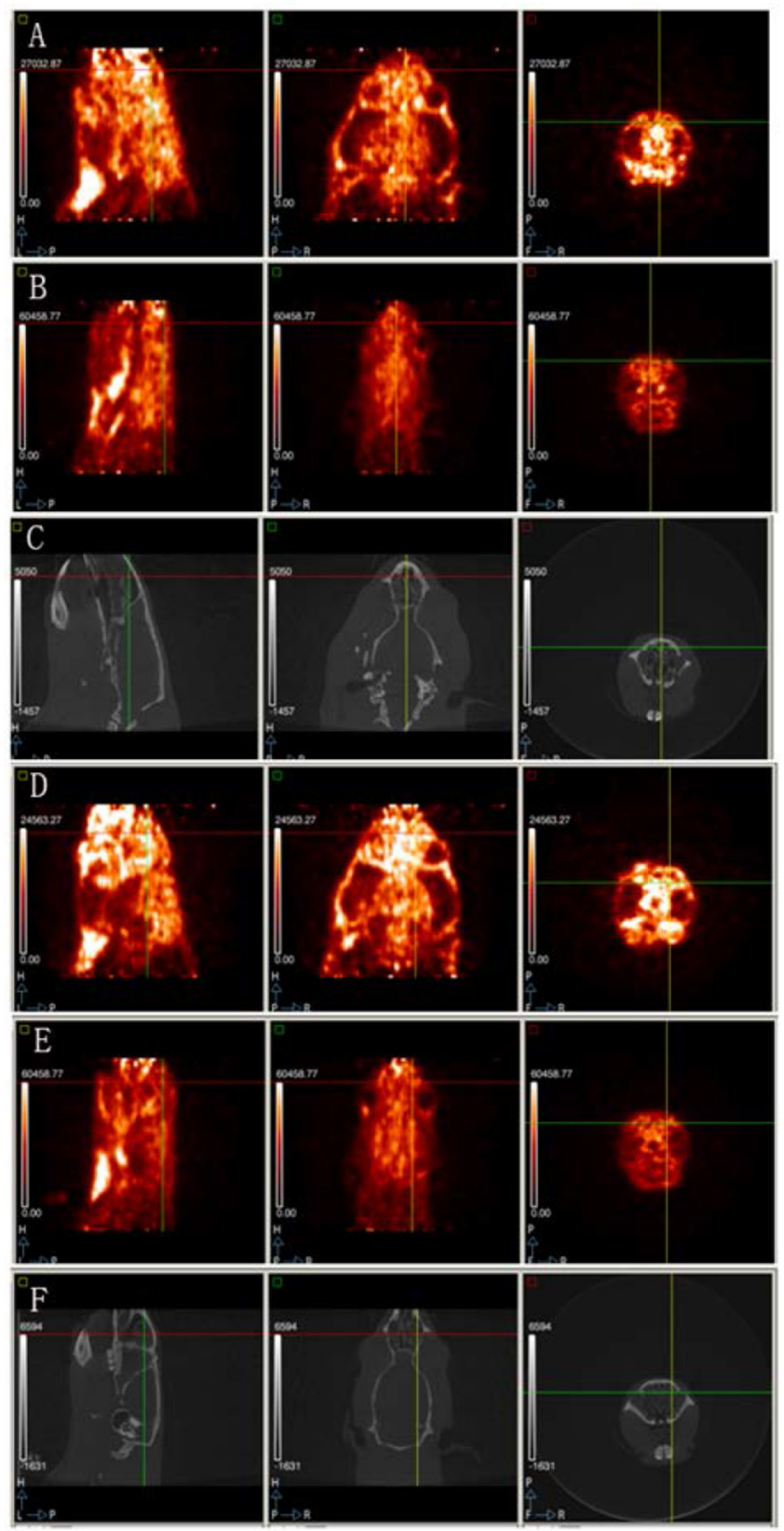

Figure 2 (A) the prefrontal cortex PET scan of the treatment group; (B) the prefrontal cortex PET scan of the control group; (C) the prefrontal cortex CT scan of the treatment group; (D) hippocampus PET scan of the treatment group; (E) hippocampus PET scan of the control group; (F) hippocampus CT scan of the control group. Each panel shows the sagittal, coronal and horizontal positions from left to right. Brighter areas reveal a higher uptake ratio in the brain. The treatment group combined more radioactive material than the control group in both brain areas. 

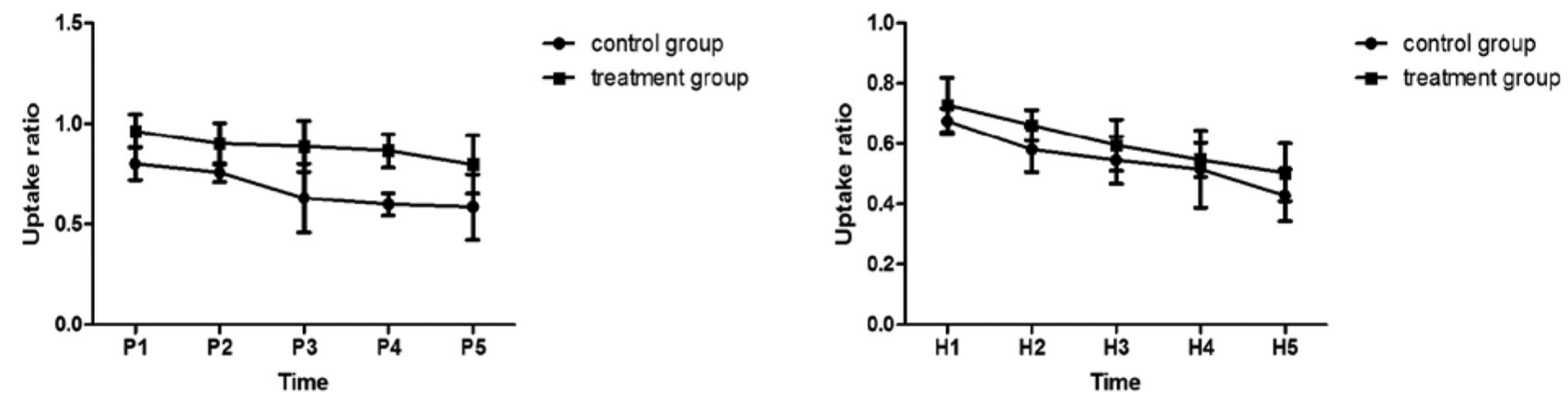

Figure $3 \mathrm{~A}:{ }^{11} \mathrm{C}-\mathrm{PK} 11195$ uptake ratio in the prefrontal cortex $\mathrm{B}:{ }^{11} \mathrm{C}-\mathrm{PK} 11195$ uptake ratio in hippocampus note: $\mathrm{p} 1-\mathrm{p} 5$ means 5 time points ${ }^{11}$ C-PK11195 uptake ratio in the prefrontal cortex; h1-h5 means 5 time points ${ }^{11}$ C-PK11195 uptake ratio in hippocampus.

\section{RESULTS}

\section{Behavioural measures}

As shown in figure 1A, the treatment group had a significantly higher locomotor activity than the control group $(t=5.239, \mathrm{p}=0.002)$.

During the passive avoidance task, the treatment group showed a significantly longer latency on the first day (T1) than the control group $(t=2.548, \mathrm{p}=0.036)$. However, there was no difference in latency on the second day $(t=-1.452$, $\mathrm{p}=0.161$ ) between the two groups. Consequently, the treatment group showed significantly lower $T$ values than the control group, reflecting their learning and memory abilities $(t=-2.618, \mathrm{p}=0.038)$ (figure 1B).

During the active avoidance task, the treatment group showed a significantly slower rate of conditioning than the control group $(t=-4.749, \mathrm{p}=0.005$ ) (figure $1 \mathrm{C}$ ).

For the PPI test, the treatment group showed significantly lower PPI values than the control group across different prepulse intensities (72dB: $t=-2.603, p=0.028 ; 74 \mathrm{~dB}$ : $t=-2.520, \mathrm{p}=0.047 ; 78 \mathrm{~dB}: t=-7.718, \mathrm{p}<0.001$ ) (figure 1D).

\section{Micro-PET/CT scan}

The isoquinoline PK11195 binds to the translocator protein $(18 \mathrm{kDa})$, the expression of which is believed to be enhanced in microglia during activation. ${ }^{2111}$ C-PK11195 uptake ratio was significantly slower in both the hippocampus $(t=-4.462, \mathrm{p}=0.001)$ and prefrontal cortex $(t=$ $-3.990, \mathrm{p}=0.003)$ in the treatment group compared with the control group (figures 2 and 3 ).

\section{Immunohistochemistry}

Most of the microglia cells in the control group were in a resting state. The PET scanning showed round or oval cell bodies (figure 4A: A-C). In the treatment group, most of the microglia cells were activated (figure 4A: B,D). There was a significant difference in the OD values of the two groups in the hippocampus and prefrontal cortex areas (hippocampus: $t=8.204, \mathrm{p}<0.001$; prefrontal: $t=6.995, \mathrm{p}<0.001$ ) (figure 4B).

\section{Correlations data}

Table 1 shows the correlations among the behavioural tests, micro-PET/CT scan and immunohistochemistry. There were significant positive correlations between the decay rate of the prefrontal cortex and PP2, PP4, PP8 in behavioural tests. There were significant negative correlations between the immunohistochemistry OD value of the prefrontal cortex brain areas and PP2, PP4, PP8 in the behavioural tests. Locomotor activity was significantly negatively correlated with the decay rate of PET/CT, and positively correlated with the OD value. Passive avoidance was significantly correlated with hippocampus area. Active avoidance was significantly negatively correlated with the OD value and positively correlated with decay rate of PET/CT (table 2). Table 3 showed a
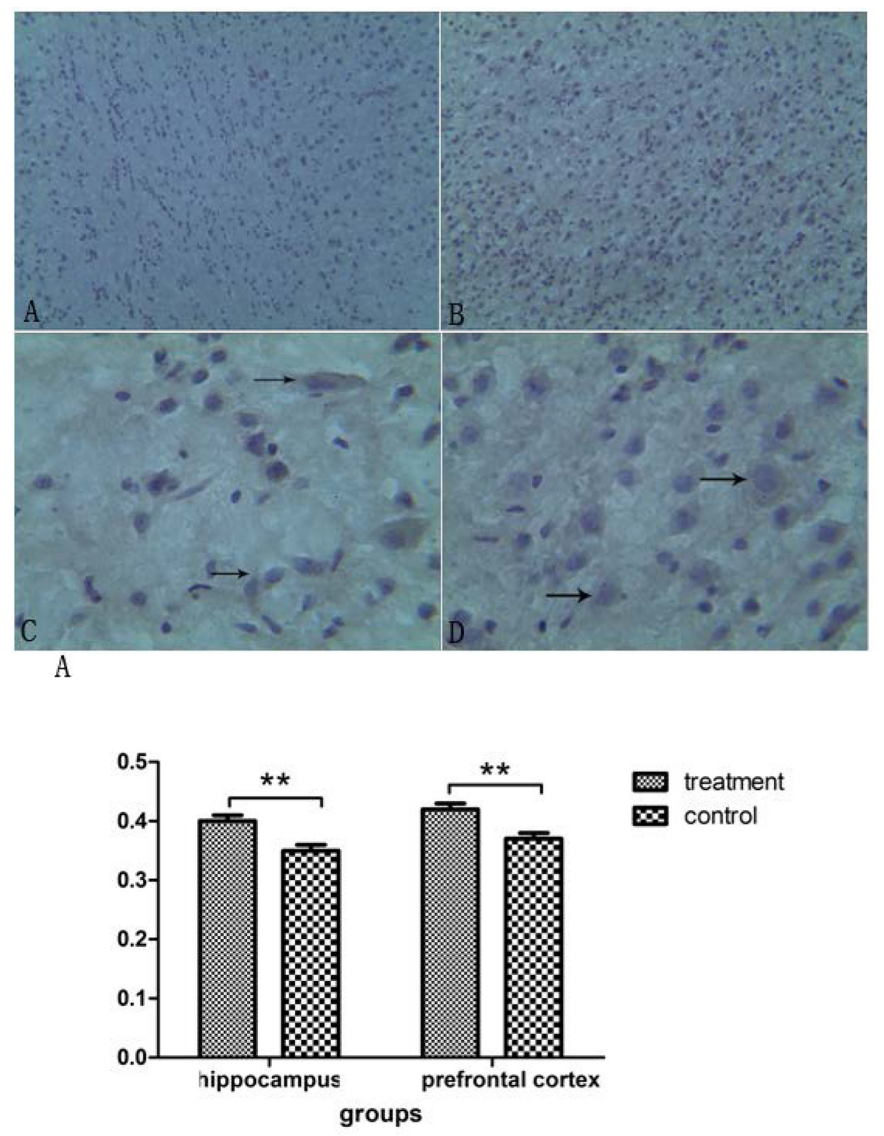

B

Figure 4 (A) CD11b-immunopositive cells in the control group $(A, C)$ and thetreatment group $(B, D)$ Group comparison of $O D$ values in the prefrontal cortex and hippocampus 
Table 2 Correlations analysis between behavioural measures and PET/CT ${ }^{11} \mathrm{C}-\mathrm{PK} 11195$ rates of decay, as well as immunohistochemistry $\mathrm{OD}$ values

\begin{tabular}{|c|c|c|c|c|}
\hline & \multicolumn{2}{|c|}{$\mathrm{PET} / \mathrm{CT}^{11} \mathrm{C}-\mathrm{PK} 11195$ uptake ratio } & \multicolumn{2}{|l|}{ OD value } \\
\hline & Prefrontal cortex & Hippocampus & Prefrontal cortex & Hippocampus \\
\hline Locomotor activity & $-0.651^{*}$ & $-0.765^{* *}$ & $0.697^{*}$ & $0.849^{* *}$ \\
\hline \multicolumn{5}{|l|}{ PPI } \\
\hline PP2 & $0.586^{*}$ & 0.534 & $-0.649^{* *}$ & -0.364 \\
\hline PP4 & $0.632^{*}$ & 0.518 & $-0.632^{*}$ & -0.567 \\
\hline PP8 & $0.750^{* *}$ & 0.439 & $-0.814^{* *}$ & -0.534 \\
\hline \multicolumn{5}{|l|}{ Passive avoidance } \\
\hline $\mathrm{T} 1$ & -0.458 & $-0.588^{*}$ & 0.450 & $0.599^{*}$ \\
\hline $\mathrm{T}$ & 0.467 & $0.598^{*}$ & -0.473 & $-0.648^{*}$ \\
\hline $\begin{array}{l}\text { Active avoidance } \\
\text { (total number of conditioned responses/500) }\end{array}$ & $0.701^{*}$ & $0.816^{* *}$ & $-0.789^{* *}$ & $-0.802^{* *}$ \\
\hline
\end{tabular}

Linear correlation analysis was used to calculatedata.

${ }^{*} \mathrm{P}<0.05$;** $\mathrm{P}<0.01$.

OD, optical density; PET,positron emission tomography; PPI, prepulse inhibition.

correlation between the micro-PET/CT scan and immunohistochemistry. There were also significantly positive correlations between the OD value and decay rate of PET/CT in the same brain area (table 3).

\section{DISCUSSION}

Main findings

This study aims to investigate microglia activation in the offspring of rats exposed to Poly I:C during pregnancy using ${ }^{11}$ C-PK11195 micro-PET/CT and immunohistochemistry. Our study demonstrated activated microglia in the prefrontal cortex and hippocampus areas, and the microglia activation was associated with SCZ-like behaviours.

We choose 56 days' offspring because: (1) the age of 56 days means a mature state of SD rats; and (2) the experimental rats in the literature are 56 days. ${ }^{7}$ In the preliminary experiment, we tried to use Iba-1 and CD11b, respectively, as the primary antibody for immunohistochemistry. The result was that $\mathrm{CD} 11 \mathrm{~b}$ was more suitable for this study.

As shown, the branches and processes of the microglia cells derived from the Poly I:C exposed SD rats were different from those of the control group. While microglia cells from the control group rats showed a more highly branched arborisation, indicative of a non-inflammatory

Table 3 Correlations analysis between PET ${ }^{11} \mathrm{C}-\mathrm{PK} 11195$ rates of decay and immunohistochemistry OD values

\begin{tabular}{cll}
\hline & \multicolumn{2}{c}{ PET $^{11}$ C-PK11195uptake ratio } \\
\cline { 2 - 3 } & Prefrontal cortex & Hippocampus \\
\hline OD value & \\
Prefrontal cortex & $-0.705^{\star}$ & -0.473 \\
Hippocampus & -0.467 & $-0.798^{* *}$ \\
\hline
\end{tabular}

Linear correlation analysis was used to calculate data.

${ }^{*} \mathrm{P}<0.05$; ${ }^{* *} \mathrm{P}<0.01$.

$\mathrm{OD}$, optical density; PET,positron emission tomography. state of microglia, the offspring from the Poly I:C-treated rats were not characterised by none or fewer branched cells, as characteristic for an activated and inflammatory or phagocytic state of microglia. ${ }^{22}{ }^{23}$ Juckel and colleagues showed that offspring microglia from Poly I:C-treated mothers were morphologically characterised by a reduced arborisation indicative for a status of higher activation compared with the offspring microglia from vehicle-treated mice. ${ }^{23} \mathrm{~A}$ reduction in ramified microglia and the overactivation of microglia cells at a young age may have a detrimental impact on the response by these animals to future neurological insults or injury. Williamson ${ }^{24}$ and colleagues suggest that adult microglia arise exclusively from the population of resident macrophages, meaning a reduction in ramified or resting microglia from an early age may have an enduring impact on the function of these resistant macrophage-like cells.

In addition, there were significant positive relationships between the results of the micro-PET/CT scan and those of the immunohistochemistry. Our findings suggest that prenatal Poly I:C-induced SCZ treatment showed active microglia in the hippocampus and prefrontal cortex brain areas. In a study of descendants of mice exposed to Poly I:C at embryonic day 9, a higher number and activation of microglia cells in the hippocampus, but not in the prefrontal cortex, existed at postnatal day $30 .{ }^{25}$ Several previous studies with C57BL/6J mice reported that active microglia cells were present in the prefrontal regions in chronic stress, and chronically available alcohol. ${ }^{26}$ The difference in the brain areas of the active microglia might be explained by the differences in the species of mice. Increasing evidence suggests a role for microglia in normal synaptic plasticity mechanisms within the adult brain, including dendritic spine retreatment and elimination. ${ }^{27}$ Some researchers have demonstrated that microglia primed as a consequence of neonatal infection and that this priming leads to aberrant 


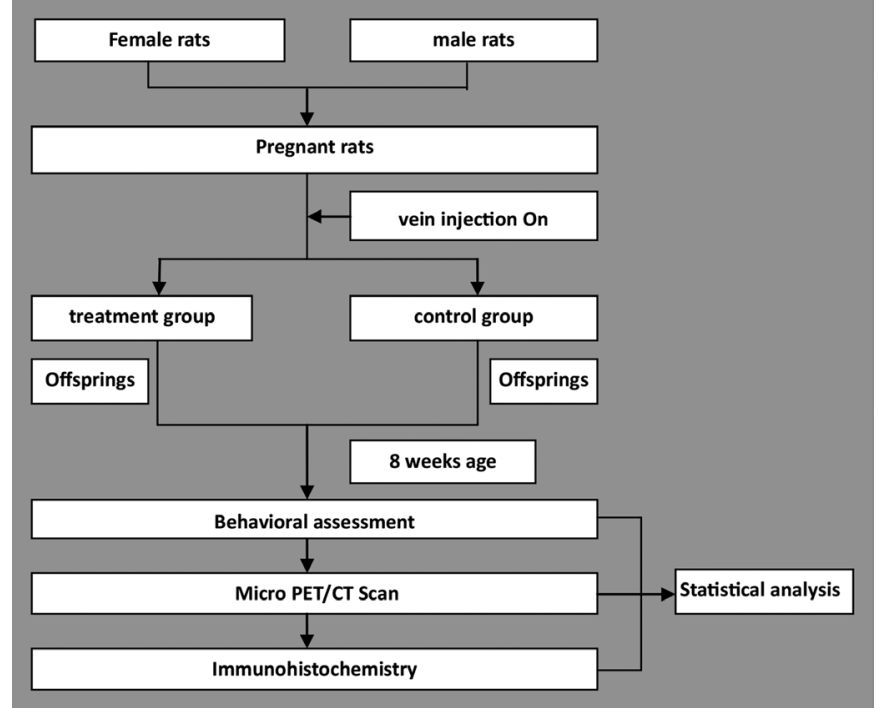

Figure 5 Flowchart of the study.

production of IL-1 specifically during learning in adulthood. ${ }^{24}$ 'Priming' of microglia, as in a fetal inflammatory challenge such as that provided by Poly I:C, may result in exaggerated response to a subsequent insult, ${ }^{28}$ due to increased production of cytokines compared with the 'unprimed' microglia. ${ }^{29}$ It is thought that this overproduction of cytokines due to a subsequent and undetected insult or infection could be the underlying mechanism leading to postnatal cognitive abnormalities related to the prefrontal cortex. ${ }^{30}$ Importantly, the resident microglia of the hippocampus are a necessary component of normal memory formation. ${ }^{24}$ Vinet and colleagues indicated that ramified microglia screen their microenvironment and additionally protect hippocampus neurons under pathological conditions. ${ }^{31}$

Our study found that the activation of the microglia was associated with SCZ-like behaviours. These findings are consistent with several previous findings. ${ }^{32-34}$ Ito and colleagues reported that changes in CA1 hippocampal c-FOS expression (an immediate early gene measure of neuronal activity) and in excitatory postsynaptic currents (measured in slices maintained in vitro) led them to conclude that the altered processing of object-place recognition was of hippocampal origin. Richetto ${ }^{34}$ and colleagues found that prenatal Poly I:C-induced immune activation led to an age-dependent decrease in GAD65 and GAD67 expression in the prefrontal cortex and hippocampus, two brain areas critically involved in (spatial) working memory. ${ }^{35}$ In a study of 16 patients with SCZ, functional connectivity indices in the left dorsolateral prefrontal cortex and the right hippocampal cortex were positively correlated with accuracy during the working memory task, while the connectivity strength in the right dorsolateral prefrontal cortex was negatively correlated with measures of the severity of the working memory task. ${ }^{37}$ Furthermore, a recent study ${ }^{38}$ found that high-risk individuals showed decreased activation compared with healthy controls in the inferior frontal and prefrontal cortex during a semantic working memory task.
In postmortem studies, activation of microglia has indeed been found in several patients with SCZ, especially in the frontal regions of brain. ${ }^{39-42}$ A previous study of 10 patients with recent-onset SCZ reported that compared with control subjects, the R- ${ }^{11} \mathrm{C}-\mathrm{PK} 11195$ binding of total grey matter was increased in patients with SCZ. ${ }^{43}$ It is possible that microglia activation may play a possible or potential role in the neuropathology of SCZ. Recently, two PET studies on SCZ versus controls showed lack of significant difference in neuroinflammation in treated patients with SCZ in the midst of a psychotic episode. The neuroinflammatory processes may take place early in disease progression or are affected by antipsychotic treatment. $^{4445}$

Compared with previous studies, the strengths of this study included the prenatal Poly I:C-induced immune activation treatment, the use of ${ }^{11} \mathrm{C}-\mathrm{PK} 11195$ as a trace of micro-PET/CT scan, combined with CD11b immunohistochemistry and behavioural assessment.

\section{Limitations}

However, the study also has several limitations: (1) it was only studied at one time point at day 56 , so it is not possible to conclude how long this activation of microglia might exist; (2) a causal relationship between microglia and cytokines cannot be drawn from the study design.

\section{Implications}

Future studies are needed to dynamically track the in vivo microglia activation from an embryonic state to adulthood in the Poly I:C prenatal infection animal model, examine the levels of cytokines in the cerebral spinal fluid and peripheral tissue and investigate the intervention strategies to prevent and treat SCZ-like behaviours.

Contributors $\mathrm{XL}$ and $\mathrm{XT}$ were responsible for the analysis and interpretation of data for this paper. All authors contributed to the writing of the paper.

Funding Funding for this study was provided by the National Natural Science Foundation of China (No 81571318 to XQS; No 81371472 to LXL; No 81401110 to $\mathrm{XL})$, the Science and Technology Planning Project of Health and Family Planning Commission (No 201501015 to XQS), the International Science and Technology Cooperation Program of Henan (No 162102410061 to XQS), the Henan Province Union Fund Project (162300410275), the Zhengzhou University doctor team project, and the Youth Fund of the First Affiliated Hospital of Zhengzhou University (to XL and LJP).

\section{Patient consent Not required.}

Open access This is an Open Access article distributed in accordance with the Creative Commons Attribution Non Commercial (CC BY-NC 4.0) license, which permits others to distribute, remix, adapt, build upon this work non-commercially, and license their derivative works on different terms, provided the original work is properly cited and the use is non-commercial. See: http://creativecommons.org/ licenses/by-nc/4.0

\section{REFERENCES}

1. Song $X Q$, Lv LX, Li WQ, et al. The interaction of nuclear factor-kappa $B$ and cytokines is associated with schizophrenia. Biol Psychiatry 2009;65:481-8.

2. Ashwood P, Krakowiak P, Hertz-Picciotto I, et al. Elevated plasma cytokines in autism spectrum disorders provide evidence of immune dysfunction and are associated with impaired behavioral outcome. Brain Behav Immun 2011;25:40-5.

3. Zhao YN, Wang F, Fan YX, et al. Activated microglia are implicated in cognitive deficits, neuronal death, and successful recovery following intermittent ethanol exposure. Behav Brain Res 2013;236:270-82. 
4. Garay PA, Hsiao EY, Patterson PH, et al. Maternal immune activation causes age- and region-specific changes in brain cytokines in offspring throughout development. Brain Behav Immun 2013;31:54-68.

5. Bland ST, Beckley JT, Young S, et al. Enduring consequences of early-life infection on glial and neural cell genesis within cognitive regions of the brain. Brain Behav Immun 2010;24:329-38.

6. Bilbo SD, Schwarz JM. Early-life programming of later-life brain and behavior: a critical role for the immune system. Front Behav Neurosci 2009;3:14.

7. Meyer U, Feldon J. To poly(l:C) or not to poly(l:C): advancing preclinical schizophrenia research through the use of prenatal immune activation models. Neuropharmacology 2012;62:1308-21.

8. Fatemi SH, Reutiman TJ, Folsom TD, et al. Maternal infection leads to abnormal gene regulation and brain atrophy in mouse offspring: implications for genesis of neurodevelopmental disorders. Schizophr Res 2008;99:56-70.

9. Meyer U, Feldon J. Neural basis of psychosis-related behaviour in the infection model of schizophrenia. Behav Brain Res 2009;204:322-34

10. Yanagi M, Southcott S, Lister J, et al. Animal models of schizophrenia emphasizing construct validity. Prog Mol Biol Trans/ Sci 2012;105:411-44.

11. Meyer U, Feldon J, Schedlowski M, et al. Immunological stress at the maternal-foetal interface: a link between neurodevelopment and adult psychopathology. Brain Behav Immun 2006;20:378-88.

12. Meyer U, Nyffeler M, Engler A, et al. The time of prenatal immune challenge determines the specificity of inflammation-mediated brain and behavioral pathology. $J$ Neurosci 2006;26:4752-62.

13. Banati RB. Visualising microglial activation in vivo. Glia 2002;40:206-17.

14. Banati RB, Newcombe J, Gunn RN, et al. The periphera benzodiazepine binding site in the brain in multiple sclerosis: quantitative in vivo imaging of microglia as a measure of disease activity. Brain 2000;123(Pt 11):2321-37.

15. Takano A. The application of PET technique for the development and evaluation of novel antipsychotics. Curr Pharm Des 2010;16:371-7.

16. Meyer U, Feldon J, Schedlowski M, et al. Towards an immunoprecipitated neurodevelopmental animal model of schizophrenia. Neurosci Biobehav Rev 2005;29:913-47.

17. Song X, Li W, Yang Y, et al. The nuclear factor-kB inhibitor pyrrolidine dithiocarbamate reduces polyinosinic-polycytidilic acid-induced immune response in pregnant rats and the behavioral defects of their adult offspring. Behav Brain Funct 2011;7:50.

18. Trigo JM, Cabrero-Castel A, Berrendero F, et al. MDMA modifies active avoidance learning and recall in mice. Psychopharmacology 2008;197:391-400

19 Wang DH, Li W, Liu XF. Chinese Medicine Formula "Jian-Pi-ZhiDong Decoction" Attenuates tourette syndrome via downregulating the expression of dopamine transporter in mice. Evid Based Complement Alternat Med 2013;2013:385685-9.

20. Ratnayake U, Quinn TA, Castillo-Melendez M, et al. Behaviour and hippocampus-specific changes in spiny mouse neonates after treatment of the mother with the viral-mimetic Poly I:C at midpregnancy. Brain Behav Immun 2012;26:1288-99.

21. Converse AK, Larsen EC, Engle JW, et al. 11C-(R)-PK11195 PET imaging of microglial activation and response to minocycline in zymosan-treated rats. J Nucl Med 2011;52:257-62.

22. Shapiro LA, Perez ZD, Foresti ML, et al. Morphological and ultrastructural features of Iba1-immunolabeled microglial cells in the hippocampal dentate gyrus. Brain Res 2009:1266:29-36.

23. Juckel G, Manitz MP, Brüne M, et al. Microglial activation in a neuroinflammational animal model of schizophrenia--a pilot study. Schizophr Res 2011;131:96-100.
24. Williamson LL, Sholar PW, Mistry RS, et al. Microglia and memory: modulation by early-life infection. J Neurosci 2011;31:15511-21.

25. Sowell ER, Thompson PM, Tessner KD, et al. Mapping continued brain growth and gray matter density reduction in dorsal frontal cortex: Inverse relationships during postadolescent brain maturation. $J$ Neurosci 2001;21:8819-29.

26. Osterndorff-Kahanek E, Ponomarev I, Blednov YA, et al. Gene expression in brain and liver produced by three different regimens of alcohol consumption in mice: comparison with immune activation. PLoS One 2013;8:e59870.

27. Tremblay MĖ, Majewska AK. A role for microglia in synaptic plasticity? Commun Integr Biol 2011;4:220-2.

28. Czeh M, Gressens P, Kaindl AM. The yin and yang of microglia. Dev Neurosci 2011;33:199-209.

29. Hagberg H, Gressens P, Mallard C. Inflammation during fetal and neonatal life: implications for neurologic and neuropsychiatric disease in children and adults. Ann Neurol 2012;71:444-57.

30. Ransohoff RM, Cardona AE. The myeloid cells of the central nervous system parenchyma. Nature 2010;468:253-62.

31. Vinet J, Weering HR, Heinrich A, et al. Neuroprotective function for ramified microglia in hippocampal excitotoxicity. $J$ Neuroinflammation 2012;9:27.

32. Barch DM, Braver TS, Carter CS, et al. CNTRICS final task selection: executive control. Schizophr Bull 2009;35:115-35.

33. Chey J, Lee J, Kim YS, et al. Spatial working memory span, delayed response and executive function in schizophrenia. Psychiatry Res 2002;110:259-71.

34. Richetto J, Calabrese F, Meyer U, et al. Prenatal versus postnatal maternal factors in the development of infection-induced working memory impairments in mice. Brain Behav Immun 2013;33:190-200.

35. Baddeley A. Working memory: looking back and looking forward. Nat Rev Neurosci 2003;4:829-39.

36. Lewis DA, Hashimoto T, Volk DW. Cortical inhibitory neurons and schizophrenia. Nat Rev Neurosci 2005:6:312-24.

37. Wolf RC, Vasic N, Sambataro F, et al. Temporally anticorrelated brain networks during working memory performance reveal aberrant prefrontal and hippocampal connectivity in patients with schizophrenia. Prog Neuropsychopharmacol Biol Psychiatry 2009;33:1464-73.

38. Broome MR, Matthiasson P, Fusar-Poli P, et al. Neural correlates of executive function and working memory in the 'at-risk mental state'. Br J Psychiatry 2009;194:25-33.

39. Steiner J, Mawrin C, Ziegeler A, et al. Distribution of HLA-DR-positive microglia in schizophrenia reflects impaired cerebral lateralization. Acta Neuropathol 2006;112:305-16.

40. Steiner J, Bielau H, Brisch R, et al. Immunological aspects in the neurobiology of suicide: elevated microglial density in schizophrenia and depression is associated with suicide. J Psychiatr Res 2008;42:151-7.

41. Radewicz K, Garey LJ, Gentleman SM, et al. Increase in HLA-DR immunoreactive microglia in frontal and temporal cortex of chronic schizophrenics. J Neuropathol Exp Neurol 2000;59:137-50.

42. Bayer TA, Buslei R, Havas $L$, et al. Evidence for activation of microglia in patients with psychiatric illnesses. Neurosci Lett 1999;271:126-8.

43. van Berckel BN, Bossong MG, Boellaard R, et al. Microglia activation in recent-onset schizophrenia: a quantitative (R)-[11C]PK11195 positron emission tomography study. Biol Psychiatry 2008;64:820-2.

44. Kenk M, Selvanathan T, Rao N, et al. Imaging neuroinflammation in gray and white matter in schizophrenia: an in-vivo PET study with [18F]-FEPPA. Schizophr Bull 2015;41:85-93.

45. Coughlin JM, Wang $Y$, Ambinder EB, et al. In vivo markers of inflammatory response in recent-onset schizophrenia: a combined study using [(11)C]DPA-713 PET and analysis of CSF and plasma. Transl Psychiatry 2016;6:e777.

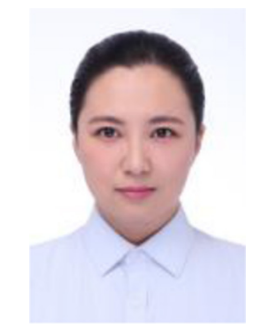

Xue Li obtained a Master degree from the First Affiliated Hospital of Zhengzhou University in July 2013. She has been working in the First Affiliated Hospital of Zhengzhou University since 2013. At present, she is working in the psychiatry department as an attending doctorand also an on-the-job doctoral student. Her research interests arebiological psychiatry and psycho pharmacology. 